\title{
Le poste et le lieu : enjeux professionnels et familiaux de la mobilité dans le corps de la magistrature en France
}

The Position and the Location: Professional and Family Issues of Mobility for Judges and Prosecutors in France

\section{Yoann Demoli et Laurent Willemez}

\section{(2) OpenEdition \\ Journals}

Édition électronique

URL : http://journals.openedition.org/travailemploi/9648

DOI : 10.4000/travailemploi.9648

ISSN : $1775-416 \mathrm{X}$

Éditeur

DARES - Ministère du Travail

Édition imprimée

Date de publication : 2 décembre 2019

Pagination : 103-130

ISSN : 0224-4365

Référence électronique

Yoann Demoli et Laurent Willemez, «Le poste et le lieu : enjeux professionnels et familiaux de la mobilité dans le corps de la magistrature en France », Travail et Emploi [En ligne], 160 | 2019, mis en ligne le 01 novembre 2020, consulté le 25 mars 2021. URL : http://journals.openedition.org/ travailemploi/9648; DOI : https://doi.org/10.4000/travailemploi.9648 


\title{
Le poste et le lieu : enjeux professionnels et familiaux de la mobilité dans le corps de la magistrature en France
}

\author{
Yoann Demoli*, Laurent Willemez.*
}

\begin{abstract}
On se représente communément la magistrature comme un corps marqué par une grande mobilité géographique et fonctionnelle (c'est-à-dire les fonctions effectivement occupées). L'objet de cet article est d'examiner cette mobilité en montrant comment elle structure le groupe et son organisation alors même qu'elle est dans les faits relativement contenue. En s'appuyant sur un traitement statistique des données de l'Annuaire de la magistrature et sur un corpus d'entretiens, cet article confirme à quel point les représentations d'une hyper-mobilité sont prégnantes, qu'elles émanent des instances de gouvernance de la profession ou des magistrats eux-mêmes. Il établit ensuite que les deux types de mobilité sont à la fois plutôt modérés et fortement genrés, dans la mesure où les hommes sont beaucoup plus mobiles que les femmes. Enfin, il met en lumière les arbitrages faits par les individus, arbitrages qui déterminent la construction de carrières différenciées selon les choix de postes dont la valeur symbolique est variable. Dans ce cadre, le genre joue un rôle prépondérant, en particulier parce qu'il contraint différemment l'articulation entre ambition professionnelle et vie domestique.
\end{abstract}

$\mathrm{O}$ n apprenait en octobre 2017 le remplacement de François Molins au poste de procureur du tribunal de grande instance (TGI) de Paris par Rémi Heitz. Ce dernier a d'abord été nommé à sa sortie de l'École nationale de la magistrature en 1989 substitut au TGI de Pontoise, avant d'être magistrat à l'administration centrale de la justice (MACJ), d'entrer en 1994 au cabinet du ministre délégué aux Relations avec l'Assemblée nationale Pascal Clément, et de retourner l'année d'après au ministère de la Justice. Il exerce ensuite quelques années en tant que procureur du TGI de Saint-Malo, puis retourne à Paris occuper un poste de vice-procureur. En 2003, après avoir été conseiller technique au cabinet du Premier ministre Jean-Pierre Raffarin, il

\footnotetext{
* Laboratoire Printemps (Université Paris-Saclay/UVSQ, CNRS) ; yoann.demoli@uvsq.fr.

** Laboratoire Printemps (Université Paris-Saclay/UVSQ, CNRS) ; laurent.willemez@ uvsq.fr.
} 
est détaché en tant que délégué interministériel à la sécurité routière, retourne en 2006 à l'administration centrale du ministère de la Justice, devient ensuite procureur au TGI de Metz, puis, jusqu'en 2015, occupe la fonction de président du TGI de Bobigny et celle de premier président de la cour d'appel de Colmar. En 2017, il retourne donc une fois encore au ministère de la Justice, comme directeur de la plus prestigieuse des directions, avant d'être nommé au poste de procureur du TGI de Paris.

Exceptionnelle par son caractère extrêmement politique et portant la marque de l'élite dirigeante de la profession, longue de onze postes et de 2185 kilomètres, une telle carrière peut donner le tournis. Néanmoins, par la succession, parfois très rapide, des postes, par le passage des frontières entre le siège et le parquet, par la distance géographique parcourue par ce magistrat, elle n'est finalement qu'un exemple, certes poussé à l'extrême, de celle de la plupart des membres de la magistrature (encadré 1). Elle illustre surtout à quel point la mobilité est au cœur des carrières du groupe et des représentations communes de celles-ci. Ainsi, magistrates et magistrats confondus, les nombres médian et moyen de postes occupés sont respectivement de 4 et 4,6.

Le groupe professionnel de la magistrature n'est pas le seul à être marqué par la mobilité : citons les énarques, l'ensemble des membres de la haute fonction publique d'État, les agents des différentes armées, ou même les cadres supérieurs réalisant des mobilités internationales. Par une recherche menée sur ces agents de l'État que sont les magistrats, à partir de l'analyse des données administratives de carrière et d'entretiens qualitatifs sur leurs trajectoires, nous revenons concrètement sur les enjeux de la mobilité.

Ces enjeux sont doubles. En premier lieu, tout comme dans les autres groupes professionnels mentionnés, la mobilité est une condition de promotion professionnelle et d'ascension dans le corps (BousSARD, 2017). Pour la magistrature néanmoins, il y a une forme de paradoxe institutionnel, voire de double contrainte. D'une part, la mobilité constitue une exigence liée à son indépendance et aux risques de corruption que constituerait le fait de rester trop longtemps dans un même poste et dans un même espace géographique ; et c'est ainsi qu'il faut comprendre la relation institutionnalisée entre nécessité de mobilité et possibilité de réussite professionnelle. Ces pratiques de mobilité obligée, qui peuvent aussi renvoyer à la valorisation du changement pour l'ensemble des salariés aujourd'hui, s'appuient surtout sur la nécessité de « dénotabilisation » de la magistrature et sont liées à la création d'une école d'application en 1945 (BOIGEOL, 1989). Mais d'autre part, les critiques sont aujourd'hui de plus en plus nombreuses à l'endroit de cette mobilité, souvent perçue comme déstabilisante dans les juridictions et critiquée car elle nuirait à l'efficacité de la justice et à la " qualité » du service public judiciaire (BREEN, 2002). Une double contrainte pèse ainsi sur les membres de la magistrature : il leur faut être mobiles, mais dans une certaine limite. Le deuxième enjeu est lié à la manière dont les individus inscrivent les mobilités dans leur carrière et, plus largement, dans leur histoire de vie et les différents espaces sociaux dans lesquels ils évoluent. La littérature montre combien la mobilité est le produit d'un certain nombre de contraintes et de ressources, et donc le résultat d'inégalités - qu'elle contribue aussi à générer. 
C'est l'objet de cet article que d'explorer ces mobilités et ces contraintes et, par voie de conséquence, les inégalités à la fois devant la mobilité et dans l'évolution des carrières magistrates. On insistera principalement sur deux types d'inégalités. La première renvoie à des questions de connaissance du corps : si les règles de mobilité et de promotion sont globalement connues, les hiérarchies symboliques entre les différents postes (qu'il s'agisse des juridictions ou des fonctions), et par conséquent les opportunités liées à telle ou telle mobilité, sont beaucoup plus difficiles à saisir. C'est pourquoi les stratégies de mobilité, c'est-à-dire « les jeux et les doubles jeux » autour du choix d'un poste ou d'un lieu plutôt qu'un autre, choix fondé sur la « connaissance pratique » de cette hiérarchie symbolique entre les postes et la valeur de ceux-ci (BOURDIEU, BOLTANSKI, 1975), méritent d'être analysées. Si la mise en visibilité de ces phénomènes de connaissance et de méconnaissance des règles juridiques et symboliques du jeu de la mobilité est indispensable, elle ne suffit pas. Nombre de recherches insistent en effet sur la manière dont les carrières professionnelles sont avant tout structurées par les inégalités de genre : les inégalités dans le travail domestique et dans les soins apportés aux enfants produisent des carrières masculines et des carrières féminines différentes. On se demandera alors si le caractère féminisé du corps (en 2017, d'après l'Annuaire de la magistrature, $66 \%$ des magistrats sont des magistrates) contribue ou non à affaiblir ces inégalités de carrière et de mobilité.

Cet article s'appuie principalement sur le traitement d'une base de données inédite et particulièrement intéressante : issue d'un fichier administratif, elle regroupe l'ensemble des carrières magistrates et leurs différentes étapes, auxquelles s'ajoutent quelques données comme le sexe, l'âge, le lieu de naissance, l'ancienneté et le mode d'accès à la magistrature ${ }^{1}$. Ces données longitudinales, qui constituent le matériau de base de la publication de l'Annuaire de la magistrature (aujourd'hui totalement dématérialisé, et présent uniquement sur l'intranet du ministère de la Justice), source des principales recherches sur la morphologie de la magistrature (BODIGUEL, 1991 ; BoIGEOL, 1998), nous ont été fournies par la Direction des services judiciaires (DSJ) du ministère de la Justice, dont la mission essentielle consiste à organiser le bon fonctionnement de toutes les juridictions judiciaires, en assurant notamment le recrutement, la nomination et la gestion de la magistrature professionnelle et des fonctionnaires des greffes. Ces données ont pourtant des limites importantes. Il y manque tout d'abord de nombreuses informations d'ordre sociodémographique, en particulier le diplôme, la profession des parents, celle du conjoint ou de la conjointe, le nombre et l'âge des enfants, etc. Manquent aussi les données de carrières antérieures pour celles et ceux

\footnotetext{
1. L'entrée dans la magistrature peut s'effectuer de plusieurs manières : le concours externe d'entrée à l'École nationale de la magistrature (ENM) est passé par les étudiants juste après leur master de droit ; le deuxième concours est réservé aux fonctionnaires de moins de 40 ans et justifiant de quatre ans de service ; enfin, le troisième concours est réservé à des candidats de moins de 40 ans justifiant de plus de huit ans d'activité dans le secteur privé. Des concours dits « complémentaires » sont en outre régulièrement organisés pour des personnes justifiant de dix ans d'expérience dans le domaine juridique. Il existe par ailleurs quelques intégrations directes dans des conditions très particulières, de même qu'un certain nombre de recrutements sur titre, dont certains sont aussi appelés « recrutements latéraux », qui ne donnent pas lieu à l'organisation d'un concours.
} 
qui ne sont pas entrés par le concours externe. Enfin, elles ne nous permettent pas de savoir comment cette mobilité est organisée à l'échelle des juridictions. Pour pallier ces absences, mais aussi pour saisir la manière dont les individus pensent les stratégies qu'ils mènent, envisagent les ressources dont ils disposent et les contraintes qu'ils vivent, nous avons construit un corpus d'entretiens biographiques auprès de juges ayant des fonctions, des trajectoires et des âges très divers $(\mathrm{N}=30)^{2}$.

Dans une première partie, nous établirons à quel point la mobilité est inscrite dans la carrière, aussi bien statistiquement que dans les représentations que chacun s'en fait. Puis, par une analyse plus fine, séparant mobilité géographique et mobilité fonctionnelle, nous nuancerons le constat répandu d'une hyper-mobilité et en exposerons les déterminants sociaux. Enfin, dans une troisième partie, nous montrerons combien la mobilité, indexée sur les positions dominantes dans la profession, renvoie à des arbitrages individuels et familiaux entre préoccupations domestiques et ambition professionnelle.

\section{ENCADRÉ 1}

\section{Promotions et mobilités dans la magistrature}

Comme pour toute la fonction publique, la mobilité et l'ascension professionnelle des magistrats et des magistrates sont organisées par des textes juridiques, qui forment un corpus de règles assez nombreuses et compliquées à saisir. Ces textes de loi, décrets ou arrêtés font par ailleurs l'objet d'interprétations et d'adaptations de la part des acteurs qui les font appliquer, qu'il s'agisse de l'instance de régulation de la profession, le Conseil supérieur de la magistrature (CSM), ou de la Direction des services judiciaires (DSJ) qui, au sein du bureau de la gestion des emplois et des carrières, guide les mobilités et les décide pour les juges, avant l'avis du CSM.

Le corps de la magistrature est réglé par l'ordonnance du 22 décembre 1958. Des décrets et des arrêtés ont permis l'application de cette ordonnance, et un certain nombre de modifications ont depuis été mises en œuvre, parmi lesquelles les plus importantes sont sans doute celles du décret du 7 janvier 1993 et de la loi organique du 8 août 2016. Pour résumer, la magistrature judiciaire est structurée en trois grades : le second grade, le premier grade et la hors hiérarchie (appelée HH). À ces grades correspondent des fonctions, des indices de traitement et des primes. Le passage d'un grade à l'autre se fait par des mobilités qui doivent suivre, elles aussi, des règles : le passage au premier grade peut ainsi intervenir à l'issue de sept ans d'ancienneté pour celles et ceux qui, sortis de l'École nationale de la magistrature (ENM), ont effectué au moins une mobilité géographique. De même, le passage à la $\mathrm{HH}$, qui correspond à des postes prestigieux dans de grandes juridictions, ne peut avoir lieu qu'après l'exercice de deux fonctions au premier grade dans deux juridictions différentes, ainsi qu'après ce que l'on appelle une mobilité statutaire

2. Les entretiens, qui ont duré entre 1 h 30 et 2 h, ont été pour la plupart réalisés avec des individus rencontrés à l'occasion de formations de « prises de postes » organisées à Paris par l'ENM. Ils ont donc été menés dans leur bureau ou dans des cafés autour de deux thématiques principales : le récit sur la carrière et les conditions de travail. 
de deux ans (c'est-à-dire un détachement dans de hautes fonctions au ministère de la Justice - directions ou sous-directions d'administration centrales -, dans des cabinets ministériels, dans d'autres administrations ou dans des entreprises publiques). Dans ce cadre, les magistrats à l'administration centrale de la justice (MACJ) constituent une classe particulière. La moitié des postes de MACJ sont en effet réservés aux membres de la magistrature sortis dans le premier tiers du classement de l'ENM. Les règles de mobilité ont donc une importance considérable, expliquée par la nécessité d'imposer aux membres du corps des règles d'impartialité et de distance face aux pouvoirs locaux.

Les nominations dans la magistrature sont publiées par décrets parus au Journal officiel $(J O)$. La nomination est assurée conjointement par le ministère de la Justice et le CSM. Le CSM émet des propositions de nomination pour les chefs de juridiction et les membres de la Cour de cassation ; les autres juges sont nommés par le ministre après avis conforme du CSM (soit un volume d'environ 400 postes). Concernant le parquet, le CSM émet un avis sur l'ensemble des nominations. Dès lors, on peut parler d'une régulation conjointe des nominations, et donc des mobilités, par le CSM et le ministère de la Justice. Plusieurs règles ont été ainsi co-construites, en particulier le fait que certaines fonctions doivent avoir des durées limitées : sept ans pour un chef de juridiction, dix ans pour les juges spécialisés ${ }^{1}$. S'il n'existe pas de règle juridique proprement dite relative à la durée minimale de l'exercice d'une fonction, une « doctrine », dite règle des deux ans, prévaut, selon laquelle la durée minimale d'exercice dans une même fonction et dans une même juridiction est de deux ans. Cette durée est portée à trois ans pour celles et ceux occupant leur premier poste ainsi que pour les chefs de cour.

Le processus de nomination passe concrètement par l'édition par la DSJ de ce qui est appelé une « transparence », c'est-à-dire la liste des postes vacants et des candidates et candidats. Un récent rapport parlementaire publié en 2017, sobrement intitulé Cinq ans pour sauver la justice!, indique que la fréquence des « transparences »s'est accrue : au-delà des deux ou trois principales transparences, on en compte généralement entre quinze et vingt tout au long de l'année (à la faveur des mouvements, notamment les départs en retraite, qui provoquent des vacances de poste et qui, une fois pourvues, entraînent à leur tour, par un jeu de chaises musicales, des vacances). Pour organiser ce «ballet», le bureau compétent au sein de la DSJ est composé d'une équipe d'une dizaine de personnes, au travail extrêmement intense dans les périodes de ce que l'on appelle les « grandes transparences $»^{2}$.

1. On appelle fonctions spécialisées les fonctions de juge d'instance, juge d'instruction, juge des enfants, juge d'application des peines, juge des libertés et de la détention (ainsi qu'en très petit nombre, juge du livre foncier). À noter que la fonction de « juge aux affaires familiales » n'est pas reconnue comme une fonction spécialisée, même si de nombreux juges exercent cette spécialité dans les tribunaux.

2. Cette procédure bureaucratique s'accompagne cependant parfois de recrutements s'appuyant sur un entretien avec le chef de juridiction. On nous a par ailleurs dit qu'il arrivait que les chefs de juridiction négocient en direct avec la DSJ l'obtention de postes. Enfin, une politique de « profilage » des postes émerge depuis quelques années. 


\section{Une description statistique et subjective des mobilités géographiques et fonctionnelles}

Au premier abord, la mobilité, envisagée comme le nombre de postes successivement occupés, apparaît particulièrement importante et structure fortement la carrière magistrate. Une analyse globale en montre en effet la prégnance dans le fonctionnement de l'ensemble du corps, mais aussi dans les représentations qu'en ont les instances de gouvernement de la profession et les membres du corps eux-mêmes.

\section{Une mobilité au cœur de la carrière magistrate}

La distribution du nombre de postes successifs occupés au cours d'une carrière, indicateur agrégeant mobilité fonctionnelle et mobilité géographique (encadré 2), indique, à première vue, une assez forte hétérogénéité des carrières magistrates (tableau 1) puisque 23,8 \% des membres du corps ont occupé un ou deux postes, $28 \%$ entre trois et quatre et $20 \%$ au moins sept (l'un d'entre eux en a même occupé dix-sept !).

Or il ressort que le nombre de postes occupés est étroitement lié à l'ancienneté dans la carrière magistrate : plus la carrière est longue, plus le nombre de postes occupés est grand (le coefficient de corrélation entre l'ancienneté dans le corps, exprimée en années, et le nombre de postes est de 0,81). De plus, lorsqu'on étudie la mobilité par sexe ou par mode d'entrée dans la profession, l'ancienneté continue de jouer un rôle central. Ainsi, les hommes ont occupé en moyenne 5 postes, contre 4,3 pour les

\section{ENCADRÉ 2}

\section{La construction des indicateurs de mobilité géographique} et de mobilité fonctionnelle

Le nombre important de juridictions ainsi que la quantité et la diversité des fonctions nécessitent la construction d'indicateurs synthétiques permettant d'appréhender les deux facettes de la mobilité, à la fois fonctionnelle et géographique.

Décrire la mobilité géographique : une géolocalisation de la base de données

Ce ne sont pas moins de 722 juridictions au sein desquelles peuvent exercer les magistrats, situées dans 412 communes différentes. Afin d'analyser les trajectoires spatiales (qui ne sont pas nécessairement des mobilités résidentielles), extrêmement diverses, des 8554 magistrates et magistrats en poste au $1^{\text {er }}$ janvier 2018, nous avons opté pour la construction d'une variable quantitative de distance parcourue entre deux postes successifs, que nous appliquons à l'ensemble de la carrière. Pour ce faire, nous avons attribué à chaque juridiction d'exercice des magistrats les coordonnées géographiques (latitude et longitude) de la commune correspondante, grâce au fichier géolocalisé des communes fourni par le site data.gouv. Nous avons alors, grâce à la formule appropriée, calculé la distance géodésique entre chaque poste $p+1$ et le poste précédent $p$. La distance parcourue entre la sortie de l'ENM et le premier poste a été calculée en prenant pour point de départ la 
commune de Bordeaux, où est sise l'ENM. Nous construisons ensuite plusieurs variables permettant de décrire la mobilité géographique :

- La distance totale additionne, pour chaque individu, les distances parcourues entre les juridictions de tous les postes occupés.

- La distance moyenne mesure, à l'échelle de chaque individu, la distance qui sépare, en moyenne, deux postes différents.

- Afin que la distance totale exprimée initialement en kilomètres ne soit pas polarisée par des valeurs aberrantes correspondant aux individus ayant voyagé dans les départements et régions d'outre-mer-collectivités d'outre-mer (DROM-COM), nous la linéarisons en en prenant le logarithme népérien (pour ne pas être contraints par les valeurs nulles de la distance, nous ajoutons un kilomètre pour chaque carrière). Nous transformons ensuite cette quantité logarithmique en score, afin de la rendre comparable au score de mobilité fonctionnelle.

Les postes en détachement, dont on ne sait rien si ce n'est la durée, ont été localisés à Paris par construction, dans la mesure où les administrations centrales concernées sont majoritairement parisiennes.

\section{Décrire la mobilité fonctionnelle : un regroupement ad hoc des fonctions}

La publication des postes vacants au $J O$ permet de dénombrer 51 fonctions qui renvoient tout aussi bien à des spécialités au siège (instruction, liberté et détention, application des peines, instance, secrétariat général, enfants, placés $^{1}$ ) qu'à des grades et à des degrés de juridiction. En revanche, elles ne correspondent pas toujours à la réalité du contentieux ; ainsi, la fonction de juge aux affaires familiales n'est pas une fonction reconnue juridiquement, même si une grande partie des juges du siège, et en particulier les femmes juges, travaillent sur le contentieux de la séparation conjugale (COLLECTIF ONZE, 2013). C'est là une des limites méthodologiques de notre travail, qu'il est aujourd'hui impossible de dépasser. Nous avons donc regroupé certaines fonctions pour les recoder, fait disparaître les grades et les juridictions (qui apparaissent dans d'autres variables) et agrégé un certain nombre de fonctions comptant peu d'individus.

Nous obtenons donc neuf modalités : magistrat exerçant en administration centrale et à l'inspection $(3,4 \%)$; chef de juridiction $(5,1 \%)$; juge d'instance $(9,8 \%)$; juge placé $(2,6 \%)$; juge des enfants $(5,4 \%)$; juge à l'application des peines $(5 \%)$; juge d'instruction $(7,1 \%)$; autre juge exerçant au siège $(39,9 \%)$; magistrat du parquet hors procureur $(21,8 \%)$. C'est sur la base de ce recodage que nous élaborons de la façon suivante un indicateur de mobilité fonctionnelle, exprimée en valeur absolue puis en valeur relative :

- Indicateur de mobilité fonctionnelle absolue : nombre de fonctions différentes occupées -1 . Cet indicateur varie de 0 à 8 et est très lié à l'ancienneté et au nombre de postes occupés.

- Indicateur de mobilité fonctionnelle relative : (indicateur de mobilité fonctionnelle absolue/nombre total de postes) $\times \mathbf{1 0 0}$. Cet indicateur permet de tenir compte $\mathrm{du}$ nombre de postes et, ainsi, de l'ancienneté dans la carrière.

1. Les juges placés sont affectés au remplacement des postes vacants de juges dans une cour d'appel, et sont susceptibles d'être mobiles au sein de cette cour, aussi bien sur le plan géographique que fonctionnel (ils restent en général quelques mois dans un poste). En contrepartie, ces juges placés sont ensuite prioritaires dans leurs choix d'affectation. 
TABLEAU 1 - Distribution du nombre de postes occupés

\begin{tabular}{lc} 
& \\
\hline Nombre de postes & Part des magistrat·es \\
\hline Entre un et deux postes & 23,8 \\
Entre trois et quatre postes & 28,0 \\
Entre cinq et six postes & 26,3 \\
Entre sept et huit postes & 14,4 \\
Entre neuf et dix postes & 5,2 \\
Plus de dix postes & 2,3 \\
\hline
\end{tabular}

Lecture : 23,8\% des membres de la magistrature ont occupé, au cours de leur carrière, entre un et deux postes.

Champ : ensemble du corps de la magistrature en poste au $1^{\text {er }}$ avril $2018(\mathrm{~N}=8554)$.

Source : Annuaire de la magistrature - traitement des auteurs.

femmes. Cet écart s'explique en partie par la différence d'âge entre les unes et les autres, l'âge moyen étant de 46,5 ans pour les femmes et de 53 ans pour les hommes en 2018. De la même façon, les lauréats du concours interne ont occupé en moyenne 4,5 postes, contre 5 pour ceux du concours externe. Autrement dit, alors que le sexe et le mode d'accès à la magistrature orientent fortement les carrières magistrates (DEMOLI, WILLEMEZ, 2018), ces facteurs ne semblent pas avoir d'effet sur le nombre de postes occupés, démontrant l'universalité du principe de l'ancienneté dans la construction de la carrière dans la magistrature.

Ce constat persiste-t-il, une fois pris en compte les différents effets de structure ? Nous proposons dans le tableau A, en annexe, une modélisation du nombre de postes, à l'aide d'une régression multiple, en fonction de six variables explicatives : le sexe, l'âge, l'ancienneté, le lieu de naissance, le mode d'accès à la magistrature ainsi qu'une interaction entre le sexe et l'ancienneté $e^{3}$.

Nos résultats montrent que l'ancienneté joue bien un rôle massif, toutes choses égales par ailleurs, dans le nombre de postes occupés. Selon notre modèle, cinq années de carrière s'accompagnent d'un poste supplémentaire $(0,2 \times 5=1)$. C'est un déterminant particulièrement important, relativement aux autres déterminants, qui sont ou non significatifs, ou d'intensité plus nuancée et ponctuelle. Être un homme plutôt qu'une femme, tout comme être plus âgé, joue un rôle négligeable dans la variation du nombre de postes occupés, ceteris paribus. Le lieu de naissance a toutefois un effet inédit sur la variable-réponse : être né en province plutôt qu'à Paris diminue le nombre de postes occupés. Enfin, on constate un faible effet positif de la variable d'interaction introduite : l'ancienneté a un effet plus marqué sur le nombre de postes occupés par les hommes que par les femmes, mais cet écart reste très faible.

3. Si les variables de sexe et d'âge permettent de rendre compte des effets de structure liés au sexe et à l'âge, c'est-àdire de montrer que les pyramides des âges sont bien différenciées selon les sexes, l'effet d'interaction vise à mettre potentiellement en lumière un effet de l'ancienneté sur le nombre de postes qui ne serait pas le même pour les hommes et pour les femmes. Sans qu'il soit possible, dans le cadre d'un modèle simple, d'identifier des effets de génération, cette interaction permet d'évaluer le bonus que représente le fait d'être un homme à l'ancienneté importante, par rapport à celui d'être une femme à la carrière de même longueur. Cette variable d'interaction, présente dans chacun des modèles de régression, a été introduite uniquement lorsque son effet était significatif. 


\section{La mobilité au cœur des représentations du corps}

Ainsi, la logique d'un corps mobile, qui semble vérifiée en première analyse et lorsque l'on agrège toutes les manières de se mouvoir en son sein, s'accompagne d'un ensemble de représentations survalorisant la mobilité. C'est le cas à la DSJ et au CSM, institutions qui passent une grande partie de leur temps à réguler ces mobilités. Mais c'est aussi le cas des membres du corps qui se font l'image d'une profession d'abord et avant tout marquée par les impératifs de mobilité permanente et les potentialités qui en découlent. Ce perpetuum mobile est à ce point ancré qu'il est défendu par ceux-là mêmes qui semblent en souffrir le plus, en l'occurrence les chefs de cour, notamment des petits et moyens TGI, que le turn-over fragilise et désorganise. Ainsi, cette procureure d'un tribunal de taille moyenne explique que la mobilité constitue l'un des axes de sa politique de gestion des magistrats :

«Et puis les encourager aussi, enfin, il faudrait impérativement... On parle de mobilité, certains y sont tenus, mais qu'un magistrat fasse carrière sans aller voir ce qui se passe ailleurs, pour moi c'est inconcevable ! Vous encouragez à l'enfermement. »

(Procureure, 55 ans, entretien du 3 octobre 2017)

Cette thématique de «l'enfermement » s'accompagne de l'expression des risques liés à l'enracinement local. Ce dernier renvoie au spectre de la notabilisation de la magistrature, à la mise sous influence, voire à la partialité ou à la corruption dont auraient fait preuve historiquement des membres du corps trop proches des élites locales (GRÉMION, 1976). Dans ces conditions, les exigences de mobilité sont perçues comme une nécessaire prise de distance avec les enjeux politiques et sociaux locaux, l'absence d'ancrage étant considérée comme la seule garantie d'une égalité de traitement entre tous les justiciables, quelle que soit leur position dans l'espace local ${ }^{4}$.

Aussi, ce n'est pas tant l'ampleur de la mobilité qui est critiquée que les procédures qui la mettent en œuvre. Les remises en question portent sur l'opacité des « transparences » (encadré 1), l'échec de certaines tentatives de mobilité ou sur les difficultés ou l'intérêt de passer d'un poste à un autre, d'une fonction à une autre, d'une région à une autre. La mobilité est défendue pour des raisons parfois particulières, pouvant aller jusqu' au tourisme, comme c'est le cas pour ce magistrat en fin de carrière :

«La chance de changer donc, elle est institutionnelle. Elle est bien pour les justiciables ; et puis aussi pour soi-même, je pense que c'est extrêmement enrichissant de changer de région. Moi, j'ai beaucoup aimé découvrir des régions nouvelles. Je suis resté de nombreuses années en Picardie dans deux postes différents. Je connaissais pas du tout cette région. C'est extrêmement attachant. J'ai beaucoup aimé Amiens ! Amiens est une ville extrêmement intéressante. Elle est connue comme étant une ville un peu triste mais en réalité, elle recèle la plus belle cathédrale de France ! [...] Elle est très agréable à habiter. »

(Parquetier, 55 ans, entretien du 27 avril 2017)

4. En cela, les exigences de mobilité peuvent être reliées aux transformations de la morphologie et de l'ethos du corps depuis les années 1970, marquées notamment par « l'autonomisation de l'univers judiciaire » (RousSEL, 2000). 
Pour d'autres, c'est plutôt les possibilités de mobilité fonctionnelle, et plus encore les occasions de multiplier les types d'activités en dehors même des juridictions par le détachement ou le passage par l'administration centrale ${ }^{5}$, qui constituent autant de chances données par l'entrée dans la carrière.

« Ça dépendra des opportunités que j'ai ou pas. Sous le statut de magistrat, les détachements, ça permet aussi de rentrer, de sortir. On est quand même un des corps de la fonction publique, à mon avis, où on a le plus de possibilités d'exercer des fonctions en interne. Parce qu' au total si on compte... moi, j'en compte au moins sept fonctions différentes dans la magistrature, sans compter l'administration centrale. Si on rajoute en plus les détachements sur le territoire national et à l'étranger, ça fait quand même beaucoup de choix. Ça fait beaucoup, beaucoup de choix. Donc je pense que tout le monde peut arriver à y trouver son compte. »

(Procureur, 40 ans, entretien du 13 décembre 2017)

Ainsi, une analyse générale de la mobilité ne nous conduit à retrouver ni la critique gestionnaire d'un mouvement permanent producteur d'inefficacité, ni la rhétorique classique de l'injonction à la polyvalence, mais une forme d'évidence et de naturalité. La mobilité est donc fortement structurée par des logiques, classiques au sein d'un système bureaucratique, de promotion à l'ancienneté, logiques qui sont appropriées de façon assez uniforme au sein de la profession. Cela ne veut pourtant pas dire qu'il n'existe pas de variations de ce phénomène lorsqu'on le considère à une échelle plus fine.

\section{Des mobilités géographique et fonctionnelle bien différenciées}

Cette mobilité généralisée ne résiste en effet pas à une analyse plus fine la décomposant. On découvre alors une mobilité beaucoup plus contenue, dont l'ampleur mesurée est en partie un artefact et est fortement reliée aux caractéristiques sociales des magistrats. Parce qu'elle maille finement le territoire métropolitain comme ultramarin et qu'elle offre une très grande diversité de fonctions, l'organisation judiciaire permet une mobilité géographique, comme fonctionnelle (cf. supra), que nous aborderons successivement. La mobilité fonctionnelle, apparemment particulièrement intense, est en réalité relativement complexe à saisir.

5. On ne reviendra pas dans cet article sur ces possibilités de mobilité que sont le détachement et l'administration centrale, qui méritent une analyse dédiée. Fortement encouragé depuis une dizaine d'années, le détachement est même devenu en 2007 obligatoire pour la plupart des nouveaux magistrats (même si le nombre de postes offert au détachement reste faible). Pour ce qui est de l'administration centrale, y occuper un poste permet de visibiliser à la fois une certaine ambition et une trajectoire ascendante pour des individus relativement jeunes (souvent dans leur deuxième ou troisième poste). Enfin, les carrières qu'on appellera «politiques », se traduisant par le passage par des cabinets ministériels dans lesquels les membres du corps sont très recherchés, mériteraient d'être étudiées. 


\section{Une mobilité géographique paradoxalement modérée et fortement genrée}

Venons-en maintenant à la mobilité géographique qui, de prime abord, apparaît particulièrement élevée. Elle suit toutefois une logique spécifique (importante en début de carrière, elle se réduit ensuite) et est fortement différenciée selon les propriétés sociales.

Les membres de la magistrature en activité au $1^{\mathrm{er}}$ janvier 2018 ont parcouru, au cours de leur carrière, une distance moyenne de 2508 kilomètres au sein du territoire, la médiane étant de 860 kilomètres. Si l'on se restreint aux magistrats n'ayant pas eu d'affectation dans les DROM-COM (encadré 2), ces statistiques diminuent : les magistrats concernés ont alors parcouru une distance moyenne de 1040 kilomètres au sein du territoire métropolitain, la médiane étant de 788 kilomètres, ce qui donne à voir une forme de polarisation opposant de (rares) hypermobiles à des individus, beaucoup plus nombreux, à la mobilité géographique moindre.

Alors que le maillage très fin du territoire judiciaire permettrait d'envisager une mobilité géographique potentiellement très élevée, nos indicateurs la décrivent donc comme contenue.

Cette mobilité géographique revêt deux spécificités : d'une part, elle est fortement différenciée selon les propriétés sociales et d'autre part, elle connaît une évolution non linéaire avec l'ancienneté. Les magistrats ont des mobilités beaucoup plus intenses que les magistrates, quel que soit l'indicateur envisagé : en conservant l'ensemble du champ, les femmes ont parcouru 2008 kilomètres en moyenne (avec une médiane de 788), contre 3496 pour les hommes (avec une médiane de 1060 ). La mobilité géographique est par ailleurs moins fortement liée à l'ancienneté, à l'inverse de ce qui est observé avec le nombre de postes : ici, le coefficient de corrélation entre les deux variables (ancienneté et kilométrage total) est assez faible $(\mathrm{R}=0,22)$. Autrement dit, si la multiplication des postes avec l'avancée dans la carrière semble une pratique généralisée, la mobilité géographique semble se faire sur des distances courtes pour une partie des magistrats. Le modèle de régression, présenté dans le tableau 2, propose une analyse toutes choses égales par ailleurs de la distance parcourue au cours de la carrière afin de confirmer les effets précédents.

Malgré ses limites, une analyse toutes choses égales par ailleurs ${ }^{6}$ montre qu'être un homme est associé à une plus grande mobilité géographique et accroît la distance parcourue de près de 400 kilomètres. La moindre mobilité des femmes au sein d'un même corps de la fonction publique est bien renseignée par la littérature sur les mobilités (BOIGEOL, 1997 ; MARRY et al., 2017) et s'explique notamment par la division sexuelle du travail domestique et familial. Néanmoins, elle se comprend aussi par la morphologie particulière du groupe des magistrats. L'homogamie stricte (avoir un.e conjoint-e dans la magistrature) étant plus fréquente chez les magistrats que chez les magistrates, les premiers bénéficient plus souvent que les secondes de l'avantage des

6. Les limites sont notamment dues au faible nombre de variables intégrées dans le modèle. 
TABLEAU 2 - Modélisation de la distance totale parcourue au cours de la carrière

\begin{tabular}{|c|c|c|c|}
\hline Variable & Coefficient & Erreur-type & Probabilité critique \\
\hline Constante & -730 & 251 & $* * *$ \\
\hline \multicolumn{4}{|l|}{ Sexe (réf. $=$ femme) } \\
\hline Homme & 393,9 & 61,9 & $* * *$ \\
\hline \multicolumn{4}{|l|}{ Mode d'accès (réf. = concours externe) } \\
\hline Concours interne & $-71,9$ & 125,5 & n.s. \\
\hline Autres modes d'accès & $-181,5$ & 135,4 & n.s. \\
\hline Ancienneté & $-9,6$ & 8,9 & n.s. \\
\hline \multicolumn{4}{|l|}{ Lieu de naissance (réf. = Île-de-France) } \\
\hline Hors Île-de-France & 247,2 & 70,8 & $* * *$ \\
\hline Nombre de postes & 262,5 & 19,1 & $* * *$ \\
\hline \multicolumn{4}{|l|}{ Poste dans les DROM-COM (réf. = non) } \\
\hline Au moins un poste dans les DROM-COM & 13393,7 & 94,1 & $* * *$ \\
\hline \multicolumn{4}{|c|}{$\begin{array}{l}\text { Notes : } \mathrm{R}^{2} \text { ajusté }=0,73 \text {. } \\
* * *, * * \text { et } * \text { renvoient à des paramètres significatifs aux seuils de } 1,5 \text { et } 10 \% ; \text { n.s. renvoie à des paramètres non significatifs. } \\
\text { Lecture : toutes choses égales par ailleurs, être un homme accroît de } 393,9 \text { kilomètres la distance parcourue au cours de la carrière } \\
\text { magistrate. } \\
\text { Champ : ensemble du corps de la magistrature en poste au } 1^{\text {er }} \text { avril } 2018(\mathrm{~N}=8554) . \\
\text { Source : Annuaire de la magistrature - traitement des auteurs. }\end{array}$} \\
\hline
\end{tabular}

mutations conjointes permises au sein de la magistrature, comme au sein de l'ensemble de la fonction publique d'État. De même, les conjoints des magistrates, fréquemment rencontrés pendant des études de droit, exercent plus souvent dans le secteur libéral ou le secteur marchand, de telle sorte que la poursuite de leur carrière contraint le champ des possibles géographiques des magistrates.

L'une des magistrates rencontrées en entretien nous explique ainsi avoir connu son futur mari sur les bancs de la faculté de droit. Lui devient avocat et elle réussit le concours de l'ENM. Au moment de choisir son premier poste, elle doit arbitrer entre une fonction qui lui plait et un lieu qui rend possible sa vie domestique : "Comme je m'étais mariée, que mon mari était avocat... il venait de trouver une collaboration intense [à Lyon]. Du coup, je cherchais plutôt dans la région Rhône-Alpes. » Elle reste plusieurs années juge dans le tribunal d'une ville de cette région, alors qu'elle habite à $80 \mathrm{~km}$ de là. Pour sa première mutation, même si elle préfère le siège, elle est contrainte de choisir un poste au parquet dans un tribunal de la commune de son domicile :

« Première grossesse, deuxième grossesse... et au moment de la deuxième grossesse, en plus, le tribunal brûle. Donc plus vraiment de lieu pour travailler, on partageait un bureau à trois. Donc là, je commençais à fatiguer et j'ai accepté de prendre un peu tout ce qui se présentait au plus près, et donc ça a été le parquet, à X [la ville de son domicile]. »

(Vice-présidente, 51 ans, entretien du 12 mars 2018) 
$\mathrm{Si}$, au cours de notre enquête, nous avons rencontré de nombreux hommes à la fois très mobiles et en couple, ayant adopté de fait une forme de célibat géographique ${ }^{7}$ (BERTAUX-Wiame, TriPIER, 2006), les magistrates qui ont des carrières fortement dispersées sur le territoire s'avèrent plus souvent célibataires ou divorcées ${ }^{8}$.

Enfin, être né·e ou avoir un·e conjoint·e né·e hors de l'Île-de-France, région bien pourvue en postes, augmente la mobilité géographique, ce qui peut notamment se comprendre dans des logiques de réinstallation dans la région d'origine, soit après le passage par l'ENM à Bordeaux, soit après un premier poste, souvent dans le Nord, dans le Nord-Est ou dans le Centre du pays. Ces retours s'expliquent par la nécessité de « recohabiter » avec les membres de son foyer, de se rapprocher de sa famille d'origine ou de s'enraciner dans un territoire. Au passage, cela suggère que cette logique d'enracinement social, qui est souvent attribuée soit à des élites politiques désireuses d'ancrage pour construire une carrière élective, soit à des classes populaires bénéficiant d'un « capital d'autochtonie » (RETIÈRE, 2003), est pertinente pour l'ensemble des groupes sociaux, ce qui vient ainsi contredire certains discours actuels sur le «nomadisme » et le goût pour la mobilité.

La trajectoire de cette magistrate, qui a grandi dans une grande ville du Nord de la France mais dont le conjoint vient du Sud-Est, et qui multiplie les postes de juriste dans diverses institutions, est à ce titre exemplaire. Entrée à l'ENM par le troisième concours, elle fait son stage dans un TGI de l'Hérault avant d'avoir son premier poste dans un petit TGI du département du Nord, où elle s'installe avec son conjoint et ses deux enfants (en tant que consultant en informatique, les contraintes de celui-ci sont moins fortes). Au bout de deux ans, elle demande un poste à Montpellier comme juge placée :

«L'enjeu pour mon mari, c'est qu'il avait un certain âge, et il n'avait pas envie de changer complètement de vie [...]. J'avais exposé la situation par le biais de mon syndicat, en disant que moi, ce que je voulais, c'était redescendre dans le Sud. [...] J'avais un peu tout demandé, balayé : Béziers, Nîmes... mais à partir du moment où j'avais mis placé [à Montpellier], c'est placé qui a été pris. »

Elle effectue une grande partie de son activité de juge placée dans un TGI de petite taille du département :

« Je n'avais pas envie de laisser complètement ma famille, donc... on a toujours fait ce choix-là : on est assez près de Montpellier, et c'est moi qui bouge. »

(Vice-présidente, 44 ans, entretien du 22 janvier 2018)

Aussi, contrairement aux représentations portées par et sur le corps, la mobilité géographique apparaît relativement contenue, et n'est pas en tout cas généralisée.

7. Le célibat géographique désigne le fait de vivre une partie du temps hors du domicile habituel de son ménage, en raison de son activité professionnelle.

8. Nos travaux rejoignent ceux de Jacqueline LAUFER (2005) sur les « cadres à potentiel » femmes et leurs « choix familiaux » souvent réalisés au détriment des stratégies de promotion. 
L'impression d'un mouvement perpétuel est sans doute d'abord due au fait que la carte judiciaire offre de nombreuses possibilités de déplacements géographiques, n'impliquant pas nécessairement une mobilité résidentielle. Ainsi, la magistrate citée ci-dessus a occupé trois postes, tout en habitant la même résidence ; en fin de compte, une fois le premier poste passé, sa mobilité géographique est restée limitée. Le maillage très fin du pays par les multiples juridictions, produisant ces « territoires de justice » que les politiques publiques ont beaucoup tenté de réformer (COMMAILLE, 2000), autorise des jeux très subtils de mobilité, opposant de façon polaire des carrières géographiques centripètes à des carrières très éclatées sur le territoire métropolitain.

On comprend alors mieux l'économie générale de cette mobilité géographique tout au long des trajectoires professionnelles : la carrière magistrate, comme un certain nombre de carrières de cadres et professions intellectuelles supérieures de la fonction publique ${ }^{9}$, se caractérise par un premier poste fortement contraint, situé le plus souvent dans les zones les moins attractives du territoire. Les professionnels qui y exercent sont jeunes et leur premier poste est souvent vécu comme un événement plus ou moins éprouvant de déracinement. La succession des postes suivants montre que la mobilité géographique diminue progressivement avant de s'accroître au terme du $6^{\mathrm{e}}$ poste (tableau 3 ). Ainsi, la médiane de la distance parcourue augmente avec le grade : 587 kilomètres pour les membres du second grade ; 949 pour ceux du premier grade et 1552 pour celles et ceux qui sont hors hiérarchie.

TABLEAU 3 - Distribution de la distance entre chaque poste, selon l'étape dans la carrière (en km)

\begin{tabular}{lcc}
\hline & Moyenne & Médiane \\
\hline Entre le $1^{\text {er }}$ et le $2^{\mathrm{e}}$ poste & 485 & 129 \\
Entre le $2^{\mathrm{e}}$ et le $3^{\mathrm{e}}$ poste & 570 & 49 \\
Entre le $3^{\mathrm{e}}$ et le $4^{\mathrm{e}}$ poste & 573 & 45 \\
Entre le $4^{\mathrm{e}}$ et le $5^{\mathrm{e}}$ poste & 592 & 45 \\
Entre le $5^{\mathrm{e}}$ et le $6^{\mathrm{e}}$ poste & 582 & 47 \\
Entre le $6^{\mathrm{e}}$ et le $7^{\mathrm{e}}$ poste & 613 & 69 \\
\hline
\end{tabular}

Lecture : entre le premier et le deuxième poste, la distance moyenne est de 485 kilomètres et la distance médiane de 129 kilomètres. Champ : ensemble du corps de la magistrature en poste au $1^{\text {er }}$ avril $2018(\mathrm{~N}=8554)$.

Source : Annuaire de la magistrature - traitement des auteurs.

\section{Une mobilité fonctionnelle relativement faible mais des spécialisations réelles dans les représentations}

Si les représentations concernant la mobilité géographique s'avèrent en grande partie fausses, il en est en partie de même pour la mobilité fonctionnelle. Là encore, nos indicateurs (encadré 2) montrent qu'elle est plus mesurée que ne le laisse entendre l'idée d'une mobilité fonctionnelle généralisée qui a toutefois une fonction sociale

9. Le travail d'Émilie BILAND (2011) montre l'image d'une mobilité assez limitée des cadres de la fonction publique territoriale. 
très forte : celle de porter le discours de l'unité du corps. Elle permet notamment de contrecarrer les tentations de séparation entre le siège et le parquet ou les logiques de spécialisation dans des contentieux particuliers. Face à ces évolutions possibles, les membres du corps mettent souvent en avant la «polyvalence » du juge, pour la défendre ou pour la critiquer. Ainsi cette magistrate, passée par des cabinets ministériels et l'administration centrale, souligne l'importance de ce débat en évoquant le contentieux des affaires familiales :

« À X, je me suis retrouvée à faire des ordonnances de non-conciliation de divorce. C'est pas compliqué mais par contre, c'est un rapport aux gens qui est complètement différent... et je trouve que c'est pas correct, c'est quand même des fonctions... Pour nous, c'est de l'abattage, pour les gens, c'est quasiment la seule fois de leur vie où ils voient un juge. Du coup, je suis très critique là-dessus. Bon, c'est un peu des débats généraux sur la polyvalence et la spécialisation. C'est-à-dire que c'est formidable de pouvoir exercer des fonctions extrêmement différentes, mais il faut être préparé, il faut être formé. »

(Vice-présidente, 51 ans, entretien du 13 novembre 2017)

Pourtant, les données montrent que l'on est là encore face à une mobilité mâ̂trisée, et d'ampleur moindre que ce qui a été observé pour la mobilité géographique. De fait, l'indice synthétique de mobilité fonctionnelle, autrement dit le nombre de postes différents, est relativement faible puisqu'il vaut 2,5 . Si l'on tient compte du nombre de postes occupés, la moyenne de l'indicateur de mobilité fonctionnelle relative (encadré 2 ) est égale à 33,9\%, et sa médiane à $38,9 \%{ }^{10}$. La distribution de cet indicateur est assez dispersée, puisque pour les $25 \%$ ayant la mobilité fonctionnelle la plus faible, l'indicateur prend une valeur nulle tandis que le troisième quartile vaut $58,3 \%$. Apparaît ainsi une forme de polarisation de la mobilité fonctionnelle, dont il s'agit de mieux comprendre les variations.

Notons tout d'abord que la mobilité fonctionnelle varie relativement peu avec l'ancienneté : ainsi, le coefficient de corrélation est de 0,23 . Nous présentons un modèle de régression qui porte sur les variations de la mobilité fonctionnelle relative (tableau 4). Outre la confirmation du faible lien entre l'ancienneté et l'augmentation de la mobilité fonctionnelle, deux autres liens sont particulièrement saillants : d'une part, être un magistrat, plutôt qu'une magistrate, ceteris paribus, conduit à avoir une mobilité fonctionnelle plus faible de 5 points ; d'autre part, être issu du concours interne, plutôt que lauréat du concours externe, augmente de 8,4 points la variableréponse. Autrement dit, à ancienneté comparable, toutes autres choses étant égales par ailleurs, les hommes connaissent une plus forte spécialisation (ou une polyvalence moindre !) que les femmes. On peut faire l'hypothèse que cette spécialisation, soutenue par une mobilité géographique plus forte, peut avoir des effets positifs sur les carrières masculines.

10. Cet indicateur varie de 0 à $100 \%$, certains n'ayant occupé qu'une seule fonction $(0 \%)$, au cours de leur carrière, d'autres ayant une fonction différente à chaque changement de poste $(100 \%)$. 
TABLEAU 4 - Modélisation de l'indicateur de mobilité fonctionnelle relative

\begin{tabular}{lccc}
\hline Variable & Coefficient & Erreur-type & Probabilité critique \\
\hline Constante & 44,3 & 2,4 & $* * *$ \\
\hline Sexe (réf. = femme) & $-4,5$ & 0,6 & $* * *$ \\
Homme & & & $* * *$ \\
\hline Mode d'accès (réf. = concours externe) & 1,4 & 1,2 & n.s. \\
Concours interne & 1,2 & 1,3 & $* * *$ \\
Autres modes d'accès & $-0,5$ & 0,1 & $* * *$ \\
\hline Ancienneté & & $-0,07$ & $* * *$ \\
\hline Âge & $-1,8$ & & \\
\hline Lieu de naissance (réf. = Île-de-France) & & 0,7 & \\
Hors Île-de-France & & &
\end{tabular}

Notes : $\mathrm{R}^{2}$ ajusté $=0,076$.

***,** et * renvoient à des paramètres significatifs aux seuils de 1,5 et $10 \%$; n.s. renvoie à des paramètres non significatifs.

Lecture : toutes choses égales par ailleurs évoquées dans le modèle, être un homme par rapport à une femme fait décroître de 4,5 points l'indicateur de mobilité fonctionnelle relative. Cet indicateur varie de 0 (pour une carrière ne comprenant qu'une seule fonction quel que soit le nombre de postes) à 100 (pour une carrière comprenant autant de postes que de fonctions).

Champ : ensemble du corps de la magistrature en poste au $1^{\text {er }}$ avril $2018(\mathrm{~N}=8554)$.

Source : Annuaire de la magistrature - traitement des auteurs.

Ces chiffres doivent cependant être maniés avec précaution, parce qu' ils sont loin de refléter la réalité du travail quotidien des magistrats. On pourrait aller jusqu'à dire que le constat de la mobilité fonctionnelle est en partie le résultat d'un artefact juridique, lié à la publication des postes au Journal officiel. Autrement dit, magistrates et, plus encore, magistrats, changent assez peu de fonction, même si finalement les juges du siège jugent plus de contentieux différents que ce que ne laisse entendre leur fonction officielle. Ainsi, ce juge d'instruction dans un petit TGI décrit sa semaine-type :

« Ça dépend... là, par exemple, je sais que j’ai pas mal de délibérés en JAF [juge aux affaires familiales] parce que j'ai des audiences : j'ai une audience de conciliation et une audience contentieux hors divorce ; en ce moment, c'est la troisième semaine du mois [...], le reste du temps, j'essaie de faire de l'instruction. Les tutelles mineurs, c'est un peu en fonction de l'arrivée des requêtes [...]. Après, il y a les procédures collectives, ce n'est vraiment que l'audience, c'est vraiment uniquement l'audience [...]. Bon, après, il y a les assises, mais là c'est pareil, ce n'est qu'un rôle d'assesseur. En correctionnelle, collégiale, pareil, c'est qu'un rôle d'assesseur [...]. Ah oui, je remplace aussi ma collègue JAP [juge de l'application des peines] quand elle n'est pas là. »

(Juge d'instruction, homme, 40 ans, entretien du 8 novembre 2017)

Même si cette mobilité fonctionnelle est finalement assez faible et sa mesure complexe, elle est présente dans le discours des membres du corps, et s'appuie en particulier sur un élément fort : celui des dispositions socialement constituées conduisant à préférer telle ou telle fonction, c'est-à-dire telle ou telle spécialité, tel ou tel contentieux, telle ou telle activité, tel ou tel type de justiciable. Parmi ces dispositions, soulignons l'importance du genre, comme le faisait Anne Boigeol (1993), ainsi que sur d'autres 
propriétés sociales, qu'elles soient liées à l'origine sociale, à la formation académique reçue à l'ENM ou « sur le tas » lors des premiers postes. Ces dispositions renvoient à chaque fois à des références, à des ethos particuliers, qui s'inscrivent dans un système de goûts et de dégoûts, qu'il n'est pas possible d'analyser ici dans son ensemble, tant il structure l'exercice de l'activité. On se contentera d'en donner un exemple parmi d'autres, l'opposition entre le siège et le parquet.

Ainsi, les individus ayant connu des postes à la fois au siège et au parquet sont minoritaires (près de 37,4\%) par rapport à celles et ceux qui n'ont connu que l'un ou l'autre (62,6 \% dont : 17,2 \% uniquement au parquet et près de 45,4 \% uniquement au siège $^{11}$ ). Le tableau 5 , croisant les trois types de carrières (siège et parquet ; parquet uniquement; siège uniquement) selon le sexe, permet de souligner le rôle du sexe dans les logiques de spécialisation. Si les carrières mixtes croissent fortement avec l'ancienneté (la part des carrières mixtes croît plus les cohortes sont anciennes), il subsiste toutefois des carrières spécialisées, dont la fréquence est particulièrement élevée pour les carrières passées uniquement au parquet compte tenu du nombre limité de postes de parquetier. Là encore, il existe une spécificité pour les hommes, dont les carrières exclusivement au parquet sont bien plus fréquentes que pour leurs collègues féminines, quelle que soit la cohorte observée : pour les magistrats des années 1980, la spécialisation au parquet s'élève à $8,3 \%$, soit quatre fois le taux des femmes. Pour

\section{TABLEAU 5 - Distribution du type de carrière selon le sexe pour les cohortes des trois décennies (années 1980, 1990 et 2000)}

\begin{tabular}{lcccc}
\hline Sexe & Siège et parquet & Parquet uniquement & Siège uniquement & Total \\
\hline \multirow{3}{*}{ Hommes } & 62,0 & Cohortes années 1980 & \\
Femmes & 48,4 & 8,3 & 29,7 & 100 \\
& & 2,0 & 49,6 & 100 \\
Hommes & 50,9 & Cohortes années 1990 & 34,5 & 100 \\
Femmes & 46,0 & 14,6 & 49,2 & 100 \\
& 41,7 & Cohortes années 2000 & & 100 \\
Hommes & 43,1 & 25,2 & 33,1 & 100 \\
Femmes & 37,3 & 11,3 & 45,6 & 100 \\
Tous magistrats & 17,2 & 45,5 &
\end{tabular}

Notes : les cohortes des années 1980 (respectivement 1990 et 2000) correspondent aux magistrats ayant pris leur premier poste dans la magistrature dans les années 1980 (respectivement 1990 et 2000).

Champ : ensemble des membres du corps de la magistrature ayant pris leur premier poste dans les années 1980, 1990 et 2000, en poste au $1^{\text {er }}$ avril $2018(\mathrm{~N}=8554)$.

Source : Annuaire de la magistrature - traitement des auteurs.

11. Ces chiffres, apparemment équilibrés, laissent penser qu'il existe des formes de spécialisation fortes : si les membres du corps occupent cinq postes en moyenne et si18 \% de ces derniers sont au parquet, alors, nous devrions avoir, sous réserve que les magistrats choisissent leur poste indépendamment du poste précédemment occupé, $0,18^{5}=0,002 \%$ des carrières au parquet seul. De la même manière, on devrait trouver $0,82^{5}=37,1 \%$ de carrières consacrées uniquement au siège, les $62 \%$ restant étant des carrières mixtes. Autrement dit, la distribution des types de carrières montre des spécialisations au parquet et, dans une moindre mesure, au siège, plus probables que ne le suggère la distribution des postes. 
les cohortes prenant leur poste dans les années 2000, les hommes sont plus du quart à avoir une carrière uniquement au parquet, contre un dixième des femmes.

La spécialisation au parquet peut en partie s'expliquer par les représentations que magistrates et magistrats se font de ces deux domaines, représentations que nos entretiens permettent de percevoir comme en partie genrées. Celles et ceux ayant fait le choix de cette spécialisation valorisent des éléments en lien notamment avec la fonction de maintien de l'ordre social, le travail d'enquête et avec une préoccupation répressive, qui les rapprochent des policier-ères et gendarmes sans pour autant s'y identifier (CoulangeOn et al., 2012). Par conséquent, c'est un rapport au droit et à la justice différent de celui des juges qui se manifeste comme dans les propos de ce jeune vice-procureur :

« Juge d'instruction, ça claque! C'est plus l'image du juge effectivement. Je me souviens très bien, $\mathrm{j}$ 'étais juge d'instruction à $\mathrm{X}, \mathrm{j}$ 'ai fait trois ans et quand $\mathrm{j}$ ' ai eu ma mutation sur le site de $\mathrm{X}$ [un grand tribunal de banlieue parisienne], $\mathrm{j}$ 'ai des enquêteurs qui m'ont dit : "C'est disciplinaire ?" Je leur disais : "Non, non, non, c'est par goût !" [...] C'est que le juge d'instruction est indépendant... mais dans le cadre que le parquet lui a fixé ! Moi, à partir du moment où on me dit que je suis libre dans un carré, je considère que je ne suis pas libre ! [...] Et puis, finalement au parquet, on a peut-être un petit peu moins de moyens juridiques mais simplement, en ouvrant le journal le matin, on décide de faire l'enquête qu'on a envie de faire ! [...] Vous êtes juge d'instruction, vous attendez que... Vous n'êtes qu'un réceptacle. Vous ne pouvez pas être à l'initiative. Moi, c'est finalement ça que je cherchais. »

(Vice-procureur, 37 ans, entretien du 19 avril 2017)

Du côté du siège, de nombreux - et, plus encore, de nombreuses - juges expliquent au cours des entretiens que jamais ils ou elles ne pourront être au parquet en raison de la spécificité du rôle qu'y endossent les magistrats, de la pression que l'activité y génère, en particulier du fait de la généralisation du traitement en temps réel des affaires (Bastard, Mouhanna, 2007). Dans les autres spécialisations, les juges manifestent des manières de penser similaires, qu'il s'agisse du «plaisir» de travailler sur la matière civile, du « goût » pour le travail avec les enfants pour les juges des enfants (PAillet, SERRE, 2013), ou encore de la " préférence » pour le travail solitaire du juge d'instruction. Pour autant, ces goûts s'articulent bien entendu avec des contraintes structurelles, elles-mêmes genrées. En particulier, «choisir » la fonction de juge et, dans les grandes juridictions, celle de juge aux affaires familiale aux conditions d'exercice souvent considérées comme moins prenantes par les juges eux-mêmes, signifie l'intégration des exigences du travail domestique dans le déroulement de la carrière professionnelle (BesSIÈRE, MiLle, 2013). 


\section{Arbitrages individuels et production de hiérarchies symboliques}

La mobilité est donc un phénomène complexe qui contraint les magistrats, et tout particulièrement les magistrates, à des arbitrages difficiles entre des aspirations qu'il leur faut essayer d'articuler, comme nous le verrons tout d'abord ; dans un deuxième temps, nous observerons qu'un tel fonctionnement produit à la fois des types de carrières distincts et des hiérarchies symboliques. Nous terminerons en montrant que ce que l'on peut appeler les « belles carrières » sont ainsi marquées par une mobilité importante.

\section{Articuler mobilité géographique et fonctionnelle}

Les mobilités découlent de « choix » et d'arbitrages complexes qui articulent, dans des proportions souvent différentes, des éléments divers : des logiques de carrière, des impératifs géographiques liés à une organisation domestique et à des choix résidentiels, des conditions de travail et des représentations que les magistrats et les magistrates se font de fonctions spécifiques, ces dernières résultant des dispositions personnelles des un.es et des autres. Au cœur de ces articulations, le genre occupe, on l'a vu, une position centrale.

Ces arbitrages sont souvent présentés dans les entretiens comme des choix rationnels, la carrière semblant le fruit du raisonnement d'un homo economicus maximisant son profit et sa satisfaction. Magistrats et magistrates reviennent longuement sur les possibilités qui étaient les leurs, leurs hésitations et les conseils pris auprès des collègues (de promotion, de stage, de syndicat, etc.). Dans ces récits, les «transparences » constituent une étape clé, et les interviewé.es se remémorent volontiers les « vœux utiles » qu'ils et elles ont pu faire, en fonction des postes possibles, de leur ambition professionnelle, de leurs impératifs domestiques et de leurs choix résidentiels. Il s'agissait de ne pas se tromper, de bien connaître les postes, les tribunaux, et de choisir en toute connaissance de cause. Dans ce cadre, les organisations syndicales jouent un rôle important, officiellement et officieusement, notamment en produisant des données permettant d'éviter des erreurs ${ }^{12}$. Ce qui n'empêche sans doute pas ces dernières de se produire, notamment quant à l'évaluation de la valeur des postes dans la hiérarchie symbolique.

Par ailleurs, s'informer ne signifie pas toujours s'intéresser à la position dans la hiérarchie, mais consiste aussi à se renseigner sur les conditions de travail dans les juridictions : atmosphère de travail, vétusté du tribunal, présence de greffiers et même

12. On trouve ainsi sur le site internet de l'Union syndicale des magistrats (USM), syndicat majoritaire dans la profession, des « fiches de juridiction » indiquant les effectifs théoriques et réels des cours, parfois des volumes d'affaires, et présentant un « tableau des distances kilométriques », réunissant les « distances et durées par la route » et les « liaisons ferroviaires ». 
parfois, comme nous l'avons entendu dans les entretiens, existence d'un parking. Tous ces éléments sont d'autant plus importants que les logiques de rigueur budgétaire et de managérialisation qui ont touché la justice ces trois dernières décennies ont dans certains cas - notamment dans les grands tribunaux de région parisienne - rendu les conditions de travail très médiocres. Il serait donc peu pertinent de quantifier les logiques de choix, qui sont multifactorielles, d'autant que la question de la montée dans la hiérarchie et de la réussite n'est finalement qu'un élément parmi d'autres. Cette sociologie de l'ambition professionnelle reste à faire, et il faudrait indexer ces arbitrages sur les différentes appartenances sociales des membres du corps.

\section{Une typologie des carrières construites sur des mobilités distinctes}

Pour clore cette exploration des mobilités magistrates, revenons-en à la sphère professionnelle afin de montrer que même si elles sont encouragées par l'institution et légitimées par ses membres, ces mobilités se traduisent par des carrières inégales et par l'existence d'une hiérarchie entre les postes. L'unité du corps, certes bien plus forte aujourd'hui que dans les années 1960, n'en reste donc pas moins traversée par des trajectoires bien différenciées, qui sont en partie le produit des mobilités.

Pour mettre en évidence cela, nous proposons une typologie des carrières, croisant mobilité fonctionnelle et mobilité géographique. Pour construire cette typologie, nous identifions chaque carrière par un couple de coordonnées, informées par les valeurs des indicateurs de distance parcourue et de mobilité fonctionnelle. Nous réalisons une classification ascendante hiérarchique, basée sur le critère de Ward ; une partition en cinq classes nous a semblé satisfaisante, au regard de l'inertie inter- et intra-classes.

Parmi les cinq classes, trois ont des fréquences relativement importantes (tableau B en annexe). La classification oppose quatre classes, avec des magistrats dont l'ancienneté dans la carrière est assez ou très élevée (classes 1,3, 4 et 5), à un groupe récemment entré dans la magistrature (classe 2, comptant $69 \%$ de magistrats ayant moins de huit ans d'ancienneté et, de façon corrélée, deux tiers de magistrats au second grade). Au sein du premier ensemble, cette classification fait apparaître une forte différenciation de la mobilité fonctionnelle, tandis que les variations de la mobilité géographique sont plus ténues, bien que polarisées.

La première classe (30,9\% des carrières) est composée d'individus avancés dans la carrière, puisque deux tiers ont au moins dix-sept ans d'ancienneté. Classe relativement plus masculine que l'ensemble de la population magistrate, elle se distingue également par son taux important d'individus (près d'un quart) ayant atteint le grade le plus élevé, celui dit de la « hors hiérarchie » $(\mathrm{HH})$. Les membres de ce groupe ont connu une assez forte mobilité géographique et une mobilité fonctionnelle plutôt contenue. Ainsi, près de $10 \%$ d'entre eux ont eu des carrières uniquement au parquet et $48,2 \%$ au siège. Privilégiant la fonction au lieu d'affectation, ce type de carrière est faite de moins de détachements et de passages par l'administration centrale que dans les autres classes. 
Nous avons ainsi rencontré une procureure de 45 ans, en milieu de carrière et ayant de grandes ambitions professionnelles (elle s'imagine très bien dans un poste de procureure générale) : après des études de droit et un bon classement de sortie à l'ENM, elle commence sa carrière dans un TGI de l'Est de la France, avant de devenir substitut non loin de là, de traverser la France d'est en ouest pour passer au premier grade, d'aller dans un tribunal du Nord de la France, de rapidement retourner dans l'Est, avant d'être nommée procureure dans le Nord-Ouest. Jusqu'à présent, toute sa carrière s'est déroulée au parquet, et en vingt ans, elle a connu six postes successifs. Vivant seule avec son fils, elle raconte longuement dans l'entretien à quel point elle se sent coupable de lui faire subir ses mobilités successives.

(Procureure, 45 ans, entretien du 11 janvier 2018)

La deuxième classe concentre les jeunes ( $26 \%$ des carrières), à la mobilité fonctionnelle très faible et à la mobilité géographique plus forte. Ils ont en effet connu en moyenne deux postes, $50 \%$ d'entre eux n'en ayant occupé qu'un seul. Un tel parcours est finalement typique des débuts de carrières qui sont certainement vouées à s'inscrire dans l'une des autres classes à l'avenir. Comme on l'a déjà dit, la mobilité géographique est très contrainte pour les individus de cette classe qui se voient souvent proposer des postes loin de leurs attaches familiales et s'efforcent donc de se réenraciner ensuite, ou au contraire d'entamer une carrière « nomade ».

La troisième classe (23,4 \% des effectifs) concerne des carrières déjà bien avancées (près de la moitié des individus ont une ancienneté dans la magistrature de plus de dix-sept ans), assez polyvalentes et avec une mobilité géographique plutôt contenue. Classe relativement féminisée, elle compte par ailleurs un taux de magistrats hors hiérarchie (HH) assez faible (moins de $10 \%$ ), au vu de l'ancienneté dans la profession. Assez fréquemment marquées par un passage en administration centrale ou par le détachement, de telles carrières sont géographiquement assez dispersées : elles sont, d'une part, concentrées en région parisienne et, d'autre part, autour de cours d'appel exerçant une force d'attraction pour des magistrats s'installant ou se réinstallant durablement dans un même lieu.

Ainsi cette magistrate, née en 1969 en Île-de-France, lauréate du concours externe, et nommée dans un tribunal d'instance en Picardie, a enchaîné les postes du siège dans le département du Nord (juge d'instruction dans deux TGI, puis juge et enfin vice-présidente, ce qui lui a permis de passer au premier grade) avant de s'installer, à l'issue de son dernier poste, dans son département de naissance.

(Source : Annuaire de la magistrature)

La quatrième classe (10,1\% de l'ensemble) ressemble fort à la classe précédente, si ce n'est que l'avancée dans la carrière est moindre, puisqu' on a affaire, majoritairement, à des individus en milieu de carrière (près des trois quarts ont entre huit et vingt-quatre ans d'ancienneté). Ayant exercé, pour les deux tiers, à la fois siège et au parquet, les magistrats de ce groupe apparaissent particulièrement polyvalents, puisque leur indice de mobilité fonctionnelle médian et moyen est le plus élevé de l'ensemble des classes. 
En revanche, la carrière au parquet seul est très rare (moins de $0,1 \%$ des individus) au sein de ce groupe. Celles et ceux ayant atteint la hors hiérarchie y sont peu nombreux.

La carrière du magistrat suivant est typique de cette classe. Né au début des années 1970 en Bretagne, entré via le concours externe à l'ENM, il prend des postes à la fois au siège et au parquet. Après avoir exercé en tant que juge d'instruction au sein de la cour d'appel de Douai sur deux postes différents, il prend un poste de juge spécialisé à la cour d'appel de Bordeaux puis occupe au sein de la même cour des fonctions au parquet, en tant que vice-procureur puis procureur.

(Source : Annuaire de la magistrature)

Enfin, la cinquième classe (9,6\% de l'effectif) se distingue par une mobilité géographique particulièrement élevée, celles et ceux y appartenant ayant parcouru près de 15000 kilomètres en moyenne au long de leur carrière. Cette mobilité géographique très forte s'explique essentiellement par le passage dans les DROM-COM, qui concerne 95,8 \% d'entre eux. C'est dans cette classe que la proportion d'hommes est la plus importante (un peu moins de la moitié), et près de $20 \%$ des magistrats sont hors hiérarchie. Bien que les carrières au parquet ainsi qu' au siège y soient nombreuses, la mobilité fonctionnelle y est beaucoup plus contenue que dans les autres classes, avec un recours fréquent au détachement.

Né en 1965 dans les Yvelines, ce magistrat occupe un premier poste dans l'ouest de la France sur une fonction de juge spécialisé, avant d'exercer au parquet dans un TGI de région parisienne, et de partir dans les DROM-COM à plusieurs reprises. Entre-temps, il sera passé par l'administration centrale et aura été détaché. Il occupe actuellement un poste de conseiller dans une grande cour d'appel.

(Source : Annuaire de la magistrature)

Ce travail de classification montre que la mobilité fonctionnelle est loin d'être uniforme. Quant à la mobilité géographique, elle se caractérise par une forme de polarisation opposant une classe hypermobile, dont presque tous les membres ont effectué un passage par les DROM-COM, à des magistrats aux mobilités géographiques plus modestes. Dans le cadre d'aspirations à une réussite professionnelle maximale, la mobilité apparaît comme une ressource centrale, en particulier pour ce qui est de la mobilité géographique qui ne constitue donc pas simplement une nécessité institutionnalisée permettant les promotions, mais qui est au cœur des stratégies d'ascension. Si de nombreux magistrats et, plus encore, de nombreuses magistrates tentent, souvent pour des raisons domestiques, de faire en sorte qu'elle ne soit pas trop importante, elle est au cœur des «belles carrières ». L'enjeu consiste donc à arbitrer entre des destinées sociales distinctes, et à éventuellement accepter, par une sorte d'amor fati ${ }^{13}$, une réussite professionnelle moindre (mais néanmoins non nulle) pour construire ou préserver une vie familiale. Il existe en effet un certain nombre de stratégies de

13. Amour du destin social, l'amor fati est un « ajustement inconscient aux probabilités associées à une structure objective de domination » (BourdiEU, 1998, p. 8). 
contournement de la mobilité, par exemple effectuer des détachements ou des passages par l'administration centrale, qui permettent le plus souvent de restreindre les mobilités à l'Île-de-France. Ce sont surtout les femmes qui sont amenées à faire ce « choix » de préserver la vie familiale, même s'il peut toutefois en aller différemment lorsque les deux conjoints sont magistrats et peuvent mener de concert des stratégies professionnelles parallèles. Ainsi les carrières les plus réussies sont celles d'hommes n'ayant guère eu à se soucier d'articuler une mobilité géographique à des préoccupations familiales, celles de femmes dont le conjoint est magistrat, celles, enfin, de femmes célibataires ou séparées ayant opté pour une vie uniquement tournée vers la réussite professionnelle.

\section{La mobilité au cœur des carrières dominantes}

Comment ces types de mobilités influencent-ils finalement la réussite et l'ascension professionnelles ? Pour saisir cela, nous recourons à une dernière régression, celle de la probabilité de passage au grade de la $\mathrm{HH}$, parmi les individus étant au moins au premier grade, en fonction des variables sociodémographiques, des indicateurs de mobilités précédemment exposés et d'autres variables caractérisant la carrière (tableau 6).

\section{TABLEAU 6 - Modélisation de l'accès à la hors hiérarchie en fonction des propriétés} et des carrières

\begin{tabular}{|c|c|c|c|}
\hline Variable & Coefficient & Erreur-type & Probabilité critique \\
\hline Constante & $-12,7$ & 0,8 & $* * *$ \\
\hline \multicolumn{4}{|l|}{ Sexe (réf. = femme) } \\
\hline Homme & 0,6 & 0,09 & $* * *$ \\
\hline \multicolumn{4}{|l|}{ Mode d'accès (réf. = concours externe) } \\
\hline Concours interne & $-0,5$ & 0,2 & $* *$ \\
\hline Autres modes d'accès & $-0,4$ & 0,3 & n.s. \\
\hline Ancienneté & 0,2 & 0,02 & $* * *$ \\
\hline Âge & 0,08 & 0,02 & $* * *$ \\
\hline \multicolumn{4}{|l|}{ Lieu de naissance (réf. = Île-de-France) } \\
\hline Hors Île-de-France & 0,06 & 0,11 & n.s. \\
\hline Au moins un poste en administration centrale & 0,9 & 0,1 & $* * *$ \\
\hline Au moins un poste en détachement & 1,1 & 0,1 & $* * *$ \\
\hline Indicateur de mobilité fonctionnelle & $-0,03$ & 0,003 & $* * *$ \\
\hline Indicateur de mobilité géographique & 0,03 & 0,004 & $* * *$ \\
\hline \multicolumn{4}{|c|}{$\begin{array}{l}\text { Notes : AIC }=3119,8 . \\
* * *, * \text { et * renvoient à des paramètres significatifs aux seuils de } 1,5 \text { et } 10 \% ; \text { n.s. renvoie à des paramètres non significatifs. } \\
\text { Lecture : toutes choses égales par ailleurs, être un homme, plutôt qu'une femme, augmente les chances d'être à la hors hiérarchie } \\
\text { Plus précisément, être un homme multiplie par exp }(0,6)=1,82 \text { les chances d'être à la hors hiérarchie, plutôt qu'au premier grade. } \\
\text { Champ : ensemble des membres du corps du premier grade et hors hiérarchie en poste au } 1^{\text {er }} \text { avril } 2018 \text {. } \\
\text { Source : Annuaire de la magistrature - traitement des auteurs }(\mathrm{N}=8554) \text {. }\end{array}$} \\
\hline
\end{tabular}


Concernant les propriétés sociales, le modèle confirme plusieurs éléments importants : tout d'abord, être un homme plutôt qu'une femme accroît la probabilité d'avoir les carrières les plus favorables, toutes choses égales par ailleurs. Ensuite, le modèle confirme que l'ancienneté est déterminante dans le passage à la hors hiérarchie : chaque année d'ancienneté supplémentaire a un effet positif sur la variable-réponse. Enfin, le concours externe reste la voie privilégiée de réussite dans la carrière magistrate, notamment à âge et sexe contrôlés, par rapport au concours interne ou aux voies parallèles d'entrée dans la magistrature. De plus, les freins et les accélérateurs propres à la mobilité sur la réussite dans la carrière peuvent être mis en lumière. Un poste à la chancellerie augmente fortement l'accès à la hors hiérarchie : ceteris paribus, être passé par l'administration centrale augmente la probabilité d'atteindre la hors hiérarchie. Cet effet tient à la fois à la constitution d'un réseau, à une connaissance plus fine des postes susceptibles d'être vacants et «payants », mais aussi au fait que, historiquement, les magistrats de l'administration centrale étaient recrutés directement parmi le premier tiers des promotions des élèves de l'ENM, sorte d'effet d'hystérèse du classement, également essentiel dans d'autres corps de la fonction publique. Le détachement joue un rôle comparable; d'où le constat apparemment paradoxal selon lequel, pour réussir dans la magistrature, il faut en sortir, au moins provisoirement ! Ce paradoxe n'est toutefois pas si fort, lorsque l'on sait qu'une partie de ces détachements ne sont qu'apparents, puisque les postes les plus élevés de la chancellerie (sous-directeur et directeur d'administration centrale) sont pourvus par détachement. Par ailleurs ces sorties provisoires peuvent aussi permettre à des magistrats de se lancer dans des carrières plus ou moins proches du monde politique, qui peut soutenir par la suite des ascensions rapides. Enfin, les effets propres des variables de mobilité sont antagonistes : toutes choses égales par ailleurs, l'indicateur de mobilité géographique est corrélé positivement et très significativement à l'accès aux positions les plus prestigieuses. À l'opposé, l'indicateur de mobilité fonctionnelle y est corrélé négativement : alors que le modèle régulièrement mis en avant est celui du juge polyvalent, la spécialisation fonctionnelle semble plutôt soutenir la réussite professionnelle, mesurée par l'accès à la hors hiérarchie.

Enfin, si l'on se concentre sur une élite d'encadrement de la profession, la description des carrières des individus ayant occupé un poste de premier président et de procureur général ${ }^{14}$ permet d'affiner de tels résultats. Le profil de ces chefs de cour d'appel confirme et même renforce les déterminants mis au jour : hommes pour les deux tiers et lauréats du concours externe pour plus de $90 \%$ d'entre eux, procureurs généraux et premiers présidents ont parcouru en moyenne 7648 kilomètres au cours de leur carrière, soit trois fois la distance couverte par la population générale ; en comparaison de cette hypermobilité géographique, leur mobilité fonctionnelle reste contenue, avec un indicateur égal en moyenne à 35 , mais leur polyvalence est importante, puisqu'aucun d'entre eux n'est passé exclusivement par le siège (quatre ont 
une carrière uniquement au parquet). Ayant été détachés pour $42 \%$ d'entre eux, ils sont près d'un tiers à être passés par l'administration centrale, tout comme dans les DROM-COM.

Cet article avait pour objectif de montrer l'intérêt d'étudier la mobilité au sein d'un groupe professionnel, et donc de saisir des carrières dans leur ensemble, au regard des propriétés sociales des personnes concernées. Cela nous semblait d'autant plus pertinent que la profession de magistrat apparaît volontiers comme un idéal-type de profession mobile et ce, alors même que l'organisation de la justice en France se caractérise par un maillage territorial dense et une moindre concentration des postes de direction en région parisienne. Nous avons essayé de mettre en évidence une réalité des mobilités plus nuancée que celle que les instances de régulation, mais aussi les membres de la profession, se représentent : non seulement parce que ces mobilités ne sont finalement pas si nombreuses mais parce qu'en outre, elles sont fortement déterminées socialement, et en particulier par le genre. Surtout, et les constats sont évidemment liés, les figures de la grande mobilité sont associées à des réussites professionnelles et à des positions dominantes dans la hiérarchie de la profession et à un passage dans une collectivité d'outremer.

L'analyse que nous proposons gagnerait à être réitérée sur d'autres groupes professionnels de la haute fonction publique, dont les représentations sont également marquées par la mobilité : par exemple, les militaires ou encore les hauts fonctionnaires, notamment les énarques (en particulier ceux exerçant au sein de « la préfectorale »). Pour ces professions, le travail sur des données longitudinales, aussi imparfaites soient-elles, permettrait de revenir sur la question de l'ambition et de la réussite professionnelles, non seulement dans son articulation avec ou son opposition à la sphère domestique, mais aussi sur ses liens avec l'ethos professionnel. Pour ce faire, tous ces éléments devraient être indexés aux dispositions des individus et, in fine, à leurs propriétés sociales, dont le genre n'est qu'un paramètre, même s'il joue à coup sûr un rôle déterminant. Une analyse de ce type permettrait aussi de montrer comment se jouent, au sein d'un couple donné, les deux parcours professionnels - perspective de travail d'autant plus intéressante au sein de la magistrature que celle-ci est marquée par une homogamie particulièrement élevée.

\section{BIBLIOGRAPHIE}

BASTARD B., Mouhanna C. (2007), Une justice dans l'urgence. Le traitement en temps réel des affaires pénales, Paris, Presses universitaires de France.

BertauX-Wiame I., TRIPIER P. (2006), « Les intermittents du foyer ou les arrangements entre membres des couples qui travaillent loin l'un de l'autre », Cahiers du genre, n ${ }^{\circ} 41$, pp. 11-22. 
Bessière C., Mille M. (2013), « Le juge est (souvent) une femme. Conceptions du métier et pratiques des magistrates et magistrats aux Affaires familiales », Sociologie du travail, vol. 55, $\mathrm{n}^{\mathrm{o}} 3$, pp. 341-368.

BILAND É. (2011), « Fonction publique territoriale : de la mobilité interne à la mobilité spatiale ? », Travail et Emploi, $\mathrm{n}^{\circ}$ 127, p. 5366.

Bodiguel J.-L. (1991), Les Magistrats, un corps sans âme ?, Paris, Presses universitaires de France.

BoIGEOL A. (1989), Histoire d'une revendication : l'École de la magistrature, 1945-1958, Vaucresson, CRIV.

BoigeOL A. (1993), « La magistrature française au féminin : entre spécificité et banalisation », Droit et Société, n ${ }^{\circ} 25$, pp. 489-523.

Boigeol A. (1997), « Les magistrates de l'ordre judiciaire : des femmes d'autorité », Cahiers du Mage, $\mathrm{n}^{\mathrm{o}} 1$, pp. 25-35.

Boigeol A. (1998), La Magistrature « hors les murs ». Analyse de la mobilité extra-professionnelle des magistrats, Paris, La Documentation française.

Bourdieu P. (1998), La Domination masculine, Paris, Seuil.

BOURDIEU P., BOLTANSKI L. (1975), « Le titre et le poste : rapports entre le système de production et le système de reproduction ", Actes de la recherche en sciences sociales, $\mathrm{n}^{\circ} 1-2, \mathrm{pp} .95-107$.

BOUSSARD V. (2017), « Les dispositifs de gestion de la mobilité des cadres : reproduction des inégalités de carrières et découplage organisationnel », in Ganem V., Lafuma E., Perrin-Joly C. (coord.) Interroger les nouvelles formes de gestion des ressources humaines : dispositifs de personnalisation, acteurs et effets, Toulouse, Octarès, pp. 49-65.

BREEN E. (dir.) (2002), Évaluer la justice, Paris, Presses universitaires de France.

Collectif OnZe (2013), Au tribunal des couples. Enquête sur des affaires familiales, Paris, Odile Jacob.

Commaille J. (2000). Territoires de justice. Une sociologie politique de la carte judiciaire, Paris, Presses universitaires de France.

Coulangeon P., Pruvost G., Roharik I. (2012), « Les idéologies professionnelles. Une analyse en classes latentes des opinions policières sur le rôle de la police », Revue française de sociologie, vol. 53, $\mathrm{n}^{\circ} 3$, pp. 493-527.

Demoli Y., Willemez L. (2018), « Les magistrats : un corps féminisé et mobile », Infostat justice, $\mathrm{n}^{\circ} 161$.

GRÉmion P. (1976), Le Pouvoir périphérique. Bureaucrates et notables dans le système politique français, Paris, Seuil.

LAUFER J. (2005), « La construction du plafond de verre : le cas des femmes cadres à potentiel », Travail et Emploi, $\mathrm{n}^{\mathrm{0}} 102$, pp. 31-44.

Marry C., Bereni L., Jacquemart A., Pochic S., Revillard A. (2017), Le Plafond de verre et l'État. La construction des inégalités de genre dans la fonction publique, Malakoff, Armand Colin. 
Le poste et le lieu : enjeux professionnels et familiaux de la mobilité dans le corps de la magistrature en France

PAillet A., Serre D. (2013), D'un juge à l'autre. Les variations de pratiques de travail chez les juges des enfants, recherche réalisée avec le soutien de la Mission de recherche Droit et justice. Retière J.-N. (2003), « Autour de l'autochtonie. Réflexions sur la notion de capital social populaire », Politix, n ${ }^{\circ}$ 63, pp. 121-143.

Roussel V. (2000), « Scandales politiques et transformation des rapports entre magistrature et politique », Droit et Société, n ${ }^{\circ}$ 44-45, pp. 13-39.

\section{ANNEXE}

TABLEAU A - Modélisation du nombre de postes en fonction de différentes propriétés des magistrats

\begin{tabular}{lccc}
\hline Variable & Coefficient & Erreur-type & Probabilité critique \\
\hline Constante & 1,96 & 0,14 & $* * *$ \\
\hline $\begin{array}{l}\text { Sexe (réf. }=\text { femme) } \\
\text { Homme }\end{array}$ & 0,17 & 0,07 & n.s. \\
\hline $\begin{array}{l}\text { Mode d'accès (réf. = concours externe) } \\
\text { Concours interne }\end{array}$ & 0,19 & 0,07 & $* * *$ \\
Autres modes d'accès & 0,01 & 0,08 & n.s. \\
\hline Ancienneté & 0,2 & 0,005 & $* * *$ \\
\hline Ancienneté $\times$ homme (réf. $=$ ancienneté $\times$ femme) & 0,01 & 0,003 & $* *$ \\
\hline Âge & $-0,01$ & 0,005 & $* * *$ \\
\hline Lieu de naissance (réf. $=$ Île-de-France) & & & $*, 04$ \\
\hline Hors Île-de-France & $-0,18$ & & $*$ \\
\hline
\end{tabular}

Notes $: \mathrm{R}^{2}$ ajusté $=0,66$.

***,** et * renvoient à des paramètres significatifs aux seuils de 1,5 et $10 \%$; n.s. renvoie à des paramètres non significatifs.

Lecture : toutes choses égales par ailleurs évoquées dans le modèle, une année d'ancienneté augmente de 0,2 le nombre moyen de postes occupés par un magistrat au cours de sa carrière. Un magistrat appartenant à la situation de référence a en moyenne 1,96 poste selon le modèle.

Champ : ensemble des membres du corps de la magistrature en poste au $1^{\text {er }}$ avril $2018(\mathrm{~N}=8554)$.

Source : Annuaire de la magistrature - traitement des auteurs. 
TABLEAU B - Description des classes obtenues par la classification ascendante hiérarchique

\begin{tabular}{|c|c|c|c|c|c|}
\hline Variable & $\begin{array}{c}\text { Classe } 1 \\
30,9\end{array}$ & $\begin{array}{c}\text { Classe } 2 \\
26,1\end{array}$ & $\begin{array}{c}\text { Classe } 3 \\
23,4\end{array}$ & $\begin{array}{c}\text { Classe } 4 \\
10,1\end{array}$ & $\begin{array}{c}\text { Classe } 5 \\
9,5\end{array}$ \\
\hline \multicolumn{6}{|l|}{ Sexe } \\
\hline Hommes & 37,3 & 31,7 & 29,7 & 22,8 & 47,7 \\
\hline Femmes & 62,7 & 68,3 & 70,3 & 77,2 & 52,3 \\
\hline \multicolumn{6}{|l|}{ Mode d'accès } \\
\hline Externe & 78,6 & 52,4 & 72,7 & 72,6 & 74,9 \\
\hline Interne & 10,3 & 9,8 & 12,8 & 12,0 & 14,6 \\
\hline Autres & 11,1 & 37,9 & 14,5 & 15,4 & 10,5 \\
\hline \multicolumn{6}{|l|}{ Ancienneté } \\
\hline Moins de 8 ans & 3,4 & 69,1 & 15,9 & 8,5 & 3,8 \\
\hline Entre 8 et 16 ans & 30,4 & 19,2 & 33,2 & 52,1 & 30,0 \\
\hline Entre 17 et 24 ans & 16,8 & 5,7 & 20,8 & 23,4 & 20,7 \\
\hline Plus de 24 ans & 49,5 & 5,9 & 30,2 & 16,0 & 45,5 \\
\hline \multicolumn{6}{|l|}{ Grade } \\
\hline Second grade & 6,3 & 67,8 & 20,3 & 13,6 & 5,9 \\
\hline Premier grade & 68,8 & 29,7 & 70,0 & 83,6 & 73,4 \\
\hline Hors hiérarchie & 24,9 & 2,5 & 9,7 & 2,8 & 20,7 \\
\hline \multicolumn{6}{|l|}{ Mobilité fonctionnelle } \\
\hline Moyenne & 30,0 & 0,1 & 46,7 & 76,9 & 45,6 \\
\hline Médiane & 29,2 & 0 & 58,3 & 77,8 & 46,7 \\
\hline \multicolumn{6}{|l|}{ Mobilité géographique (en km) } \\
\hline Moyenne & 1177 & 1161 & 1027 & 973 & 15743 \\
\hline Médiane & 961 & 601 & 857 & 829 & 14546 \\
\hline \multicolumn{6}{|l|}{ Nombre de postes } \\
\hline Moyenne & 5,9 & 2 & 5 & 4,6 & 6,2 \\
\hline Médiane & 6 & 1 & 5 & 5 & 6 \\
\hline Passage par l'administration centrale & 12,9 & 0 & 22,1 & 23,2 & 13,7 \\
\hline Passage par le détachement & 12,2 & 0 & 16,7 & 14,4 & 17,0 \\
\hline Passage par les DOM-COM & 0,2 & 5,2 & 0,1 & 0,1 & 95,8 \\
\hline
\end{tabular}

* À l'exception des lignes « Mobilité géographique » et « Nombre de postes ».

Champ : ensemble du corps de la magistrature en poste au $1^{\text {er }}$ avril $2018(\mathrm{~N}=8554)$.

Source : Annuaire de la magistrature - traitement des auteurs. 